Anno 35, 2020 / Fascicolo 1 / p. 32-45 - www.rivista-incontri.nl - http://doi.org/10.18352/incontri.10332 (c) The author(s) - Content is licensed under a Creative Commons Attribution 3.0 Unported License Publisher: Werkgroep Italië Studies, supported by Utrecht University Library Open Access Journals

\title{
La facezia nel tardo '400, forma popolareggiante o raffinato genere umanistico? Sondaggi su un capitolo minore della produzione "letteraria" di Leonardo da Vinci
}

\section{Cristiano Amendola}

Sommersi per secoli sotto una fitta coltre di appunti, frammisti a reperti di ben altro valore, tra le carte di Leonardo si ritrovano, grazie all'intenso e ormai secolare lavoro di scavo e di riordino condotto dagli studiosi sui suoi codici, alcuni bozzetti narrativi dal sapore comico e dalla tematica spesso lasciva $i$ quali, per la levitas che li contraddistingue, oltre che per la loro evidente destinazione sociale, consentono di ricalibrare in un senso più autenticamente mondano quell'idea del genio solitario e stravagante che ancora oggi sembra caratterizzare la figura del grande artista toscano nell'immaginario collettivo. Scenette ridicole, frammenti di dialogo e narrazioni dall'andamento spesso scorciato e dal soggetto talvolta osceno sono accomunati, nelle carte leonardesche, oltre che dalla condivisione di uno stesso registro espressivo, anche dalla consimile partecipazione a un medesimo meccanismo narrativo: la risoluzione, cioè, delle tensioni narrative (minime) in un calembour finale che, intervenendo a suggellare il testo, conferisce a esso compattezza e unità. Tutto ciò permette di ricondurre agevolmente questi ioca entro l'alveo di un ben distinto genere della variegata tradizione della narratio brevis romanza: ${ }^{1}$ quello, cioè, della facezia. ${ }^{2}$

Al benemerito lavoro degli indagatori dei codici vinciani, cui, come si è detto, si deve la riscoperta di questi divertenti tasselli narrativi, si è affiancata negli anni, quando non sovrapposta, l'attività di tanti antologisti che, ormai da oltre un secolo, sono impegnati nell'ardua quanto necessaria impresa di conferire ordine e organicità a ciò che era apparso, fin dai primi sondaggi, un insieme disomogeneo di appunti di difficilissima decifrazione. Talvolta, però, ciò ha prodotto, come inevitabile controindicazione, lo sradicamento del frammento dal contesto della singola carta che, specialmente negli zibaldoni dell'artista, rappresenta una vera e propria unità semiotica, ${ }^{3}$ obliterando così parzialmente quella 'estemporaneità' che costituisce una

\footnotetext{
${ }^{1}$ Quella della narrativa breve romanza è una tradizione ricca e molto antica, che vanta al suo interno forme diversissime tra loro per prestigio, impianto retorico, cura formale. Per un profilo generale in una prospettiva narratologica si rinvia al fondamentale H.R. Jauss, 'I generi minori del discorso esemplare come sistema di comunicazione letteraria’, in: M. Picone (a cura di), Il racconto, Bologna, Il Mulino, 1985, pp. 53-72.

2 Per quanto riguarda il testo delle facezie leonardesche, ci serviremo di quello stabilito in L. da Vinci, Favole e profezie. Scritti letterari, G. Cirnigliaro e C. Vecce (a cura di), Milano, Garzanti, 2019, pp. 2130. Per la numerazione delle stesse, così come per le sigle dei codici vinciani, ci riferiremo a quelle adottate in detta edizione.

${ }^{3}$ C. Vecce, “"Scritti” di Leonardo da Vinci', in: A. Asor Rosa (a cura di), Letteratura italiana. Le Opere, vol. II, Dal Cinquecento all'Ottocento, Torino, Einaudi, 1993, pp. 109-114.
} 
delle dimensioni principali nelle quali si dà il genere della facezia: forma letteraria, certo, ma anche e forse soprattutto performance retorica e sociale, frammento estratto dalla conversazione quotidiana e fissato su carta perché di esso più efficacemente se ne potesse conservare il ricordo.

Tenendo conto di tale natura sociale e performativa della facezia, e attraverso una ricognizione del complesso processo di legittimazione di una forma che, in epoca umanistica, poteva rapidamente risalire numerosi gradini sulla scala delle gerarchie dei valori letterari, si tenterà in queste pagine di fornire un contributo teso a ricollocare questo capitolo della produzione culturale del maestro toscano entro l'ambito di una più ampia riflessione su un genere che, a dispetto della scarsa considerazione critica di cui ancora oggi sembra talvolta godere, si rivelerà tutt'altro che secondario nel contesto delle pratiche discorsive, sociali e culturali della raffinata vita cortigiana del secondo Quattrocento.

Narratio brevis e retorica umanistica nelle pratiche della conversazione cortigiana Ai tempi di Leonardo, la facezia non era argomento di quelli sui quali si potesse scherzare troppo. 0, almeno, certamente non lo era nell'ambito di quei circoli intellettuali gravitanti in quegli anni attorno alla corte di Ludovico il Moro, ${ }^{4}$ presso $i$ quali, durante il suo lungo soggiorno milanese, lo stesso Leonardo sembrò a più riprese volersi accreditare. ${ }^{5}$ Nei testi chiave sui quali si fondarono le loro aspirazioni di rinascenza, - principalmente l'Etica nicomachea, il De oratore e l'Institutio oratoria -, ${ }^{6}$ gli umanisti poterono ritrovare un'articolata riflessione sul comico che, riadattata al mutato contesto culturale, permise loro di restituire lustro alle forme della narratio brevis medievale - tra le quali, e principalmente, la facezia. Con uno scatto dialettico e costruttivo che sempre caratterizzerà gli orientamenti del loro rapporto con i classici, essi, inoltre, se ne servirono per formulare una moderna teoria della conversazione faceta che, nell'esito ultimo e più alto rappresentato dal De sermone di Giovanni Pontano, condurrà a una effettiva estensione degli ambiti di pertinenza della retorica in direzione di una regolamentazione di un aspetto della vita associata da sempre in essi negletta: quello, cioè, delle relazioni linguistiche nei

\footnotetext{
${ }^{4}$ Sulla facezia umanistica molto si è scritto negli anni; mi limito pertanto a segnalare qui soltanto quei contributi che, a mio parere, più risultano utili a centrare i termini della questione affrontata in questo scritto: G. Ferroni, 'La teoria classicistica della facezia da Pontano a Castiglione', in: Sigma, XIII, 2-3 (1980), pp. 69-96; G. Alfano, 'La misura e lo scacco: sul “De Sermone” di Gioviano Pontano', in: Modern Languages Notes, 115, 1 (2000), pp. 13-33; A. Quondam, La conversazione: un modello italiano, Roma, Donzelli, 2007; F. Tateo, 'Percorsi della facezia tra Tre e Quattrocento', in: InVerbis. Lingue Letterature Culture, 2 (2011), pp. 59-70; E. Curti, 'Le facezie umanistiche', in: E. Menetti (a cura di), Le forme brevi della narrativa, Roma, Carocci, 2019, pp. 63-79. Sul ruolo della facezia nella riflessione pedagogica umanistica utile, ancora, è il saggio di L. Salvarani, 'L'altra paideia. Libri di motti, facezie e "detti piacevoli” nell'Italia del Rinascimento', in: Rassegna di Pedagogia, LXXIV, 1-2 (2016), pp. 123-137.

${ }^{5}$ A una partecipazione di Leonardo ai circoli cortigiani della Milano di Ludovico il Moro sin dai primi anni del suo arrivo in città sembra alludere Bernardo Bellincioni in un capitolo in terza rima risalente al 1485 ca. In un sonetto del 1488, ancora il Bellincioni lo menziona tra quatro homini famosi nutriti sotto a l'umbra del Moro, in compagnia dell'umanista Giorgio Merula, dell'orefice Cristoforo Foppa, e del bombardiere Giannino Alberghetti. Su questi componimenti vd. C. Vecce, Leonardo, Roma, Salerno editrice, 2006 (I ed. Roma, 1998), pp. 87-88 e p. 107. Sui rapporti di Leonardo con l'umanista Piattino Piatti, autore di due epigrammi in latino in suo onore, si veda ancora Ivi, pp. 110-111. Sulle tormentate relazioni tra Leonardo, omo sanza lettere, e i colti scrittori di corte vd. infra.

${ }^{6} \mathrm{Nel}$ dettaglio, i passaggi dedicati al comico si leggono in: Aristotele, Etica Nicomachea, II, 7, e IV, 7-8; Cicerone, De oratore, II, § 235-289 (sezione nota anche come Excursus de ridiculis); Quintiliano, Institutio oratoria, vol. I, VI, 3 (paragrafo noto anche con il titolo De risu). Sulle teorie classiche del riso si vedano N. Ordine, Teoria della novella e teoria del riso nel Cinquecento, Napoli, Liguori, 2009 (segn. alle pp. 325); e M. Beard, Ridere nell'antica Roma, Roma, Carocci, 2016. Sul ruolo decisivo di quei testi nello sviluppo del pensiero umanistico si veda A. Battistini \& E. Raimondi, Le figure della retorica: una storia letteraria italiana, Torino, Einaudi, 1990, pp. 75-96.
} 
contesti di bassa formalità. ${ }^{7}$ Celebrata da quegli intellettuali come una virtù da coltivarsi al lume dello studio dei classici, le facetudo, neologismo coniato dal Pontano per indicare la capacità di conversare in modo piacevole e faceto, verrà riproposta, nel corso del '400, quale correlativo privato delle qualità oratorie e diplomatiche dei suoi detentori; e, come tale, essa verrà regolarmente associata, nei moderni ritratti de viris illustribus, a signori e principi umanisti di primissimo piano. ${ }^{8}$

Certo, l'aggancio della facezia alla retorica non giunse al ' 400 inaspettato. Alle spalle di quel fortunato e fecondo connubio vi fu infatti un lungo e complesso percorso, i cui primi passi si possono già intravedere in un'opera di epoca medievale, il Novellino, una raccolta di novelle e facezie messa insieme da un anonimo compilatore verso la fine del XIII secolo. ${ }^{9}$ Alla facezia, ancora, nella sua duplice funzione di liberare il locutore da una situazione tesa o potenzialmente aggressiva denotandolo, al contempo, come arguto et ingegnoso, insieme a quella di "mordere" elegantemente l'avversario ribaltandone il punto di vista attraverso un giro di parole sagace e acuto, il Boccaccio aveva dedicato, come è noto, parte delle novelle della prima giornata del Decameron, e tutte quelle della sesta. ${ }^{10}$

Nel processo di definizione di una moderna teoria classicistica della facezia, tuttavia, fondamentale fu il ruolo svolto dai Rerum memorandarum libri di Francesco Petrarca, un testo che promosse una riconsiderazione di quel genere secondo categorie storiche ed estetiche di fatto già pienamente umanistiche. ${ }^{11}$ Nelle pagine De facetiis ac salibus illustrium, esplicitamente ispirate all'Excursus de ridiculis ciceroniano per

${ }^{7}$ Cfr. G. Pontano, De sermone, E. Mantovani (a cura di), Roma, Carocci, 2002.

8 Numerose erano, a esempio, le facezie attribuite a personaggi del calibro di Cosimo de' Medici, Francesco Sforza, Federico da Montefeltro nelle raccolte del Poliziano e di Ludovico Carbone. A questo aspetto della personalità del re di Napoli Alfonso d'Aragona il biografo Antonio Panormita dedicava numerose pagine. E così anche l'umanista Galeotto Marzio, nella biografia da lui offerta al re ungherese Mattia Corvino. Cfr. risp. A. Poliziano, Detti piacevoli, T. Zanato (a cura di), Roma, Istituto della Enciclopedia Italiana fondata da Giovanni Treccani, 1983; L. Carbone, Centotrenta novelle o facezie, A. Salza (a cura di), Livorno, Giusti, 1900; A. Panormita, De dictis et factis Alphonsi regis, Vuitebergae, Typis haeredum loannis Cratonis, 1585; G. Marzio, Detti e fatti insigni di Mattia Corvino re d'Ungheria, G. Moro (a cura di), Lugano, Agorà, 2016. Sulla teoria pontaniana della facetudo si rimanda all'ottimo saggio di A. Mantovani che apre la sua ed. di Pontano, De sermone, cit., pp. 7-53.

9 Il tentativo dell'autore di quel florilegio, il cui obiettivo era quello di offrire ai lettori un catalogo di 'moduli' da riprendere in occasione di piacevoli conversazioni cortesi, si iscriveva nel contesto delle pratiche discorsive agonistiche tipiche del mondo comunale, pratiche che diedero avvio a una rilettura in senso civile della retorica classica. La brevità sulla quale si fondava l'efficacia della comunicazione secondo la Rhetorica ad Herennium era infatti individuata quale principio di riferimento stilistico della facezia, che vedeva così ridefinite le proprie forme proprio secondo le prescrizioni relative alla narratio dell'orazione. Cfr. S. Battaglia, 'Premesse per una valutazione retorica del "Novellino"', in: Filologia romanza, 6 (1955), pp. 259-286 e A. Paolella, Retorica e racconto. Argomentazione e finzione nel Novellino, Napoli, Liguori, 1987.

10 Filtrando attraverso la teoria dell'umorismo di Quintiliano alcune delle conversazioni presenti nel Centonovelle, lo scrittore certaldese inaugurava quella che con giusta enfasi è stata definita 'la conquista di un realismo umanistico'. L'osservazione si legge in Carlo Muscetta, Giovanni Boccaccio, Roma-Bari, Laterza, 1974, p. 309. Con la celebre novella VI, 5, che vedeva come protagonisti Giotto e Forese da Rabatta, inoltre, Boccaccio dava avvio al mito dell'artista arguto e faceto, mito destinato ad alimentarne la rappresentazione in gran parte delle biografie dei due secoli successivi. Sul rapporto tra novellistica e biografia en artiste vd. anche G. Tanturli, 'Le biografie d'artisti prima del Vasari', in: Il Vasari storiografo e artista. Atti del Congresso internazionale nel IV centenario della morte. Arezzo-Firenze, 2-8 settembre 1974, Firenze, Istituto nazionale di studi sul Rinascimento, 1976, pp. 275-298; L. Riccò, 'Tipologia novellistica degli artisti vasariani', in: G. C. Garfagnini (a cura di), Giorgio Vasari tra decorazione ambientale e storiografia artistica. Atti del convegno di Arezzo 1981, Firenze, Olschki, 1985, pp. 95-115 e M. Zaccarello, 'Ingegno naturale e cultura materiale: i motti degli artisti nel "Trecento Novelle" di Sacchetti', in: Italianistica, 38, 2 (2009), pp. 129-140.

11 F. Petrarca, Rerum memorandarum libri, M. Petoletti (a cura di), Firenze, Le lettere, 2014. Sulla prospettiva storica alla base del testo petrarchesco cfr. P. Cherchi, 'Petrarca, Valerio Massimo e le “concordanze delle storie"', in: Rinascimento, 42 (2002), pp. 31-65. 
quanto riguardava la classificazione tipologica della facezia, ${ }^{12}$ infatti, essa non soltanto appariva per la prima volta come forma praticabile in quanto legittimata dall'esempio degli antichi: secondo quelle pagine, i moderni potevano ora finanche gareggiare con essi, quando non addirittura superarli nell'esercizio stesso di quell'arte. ${ }^{13} \mathrm{Da}$ tale punto di vista, la facezia moderna veniva riconosciuta quale genere a sua volta degno di imitazione, e la sua lunga tradizione presentata quale bagaglio di tópoi cui attingere per rimodulare in maniera conveniente i registri della scrittura comica.

Innalzata a repertorio di forme degne di imitatio, la tradizione della narratio brevis medievale arrivava così a Poggio Bracciolini, autore di una celebre raccolta presente, come vedremo meglio a breve, anche nella biblioteca personale di Leonardo da Vinci, come una sorta di serbatoio di loci cui riferirsi per aggiornare il latino coevo nei registri del quotidianus. ${ }^{14}$ La lunga distanza temporale, sosteneva il Bracciolini nella praefatio del suo Liber, aveva infatti reso il latino classico incapace di significare efficacemente la realtà contemporanea. ${ }^{15}$ Per uscire da questa impasse, aggiungeva lo scrittore, non restava altro che provare a condurre il principio creativo dell'imitatio fin quasi oltre i limiti del mimetismo: solo riplasmando le parole sulle cose del mondo attraverso la traduzione in latino della facezia contemporanea, infatti, diveniva nuovamente possibile imitare efficacemente gli antichi nella descrizione del quotidiano a essi coevo.

Se, sul piano letterario, la pratica della facezia risultava dunque legittimata dall'esempio degli antichi, su quello etico era invece la teoria aristotelica del "riso ristoratore" a offrire ora lo spunto per l'attribuzione al genere faceto di una precisa funzionalità sociale: la conversazione piacevole e gli scherzi linguistici dovevano infatti servire a ritemprare lo spirito degli astanti durante i momenti di sospensione delle attività lavorative, quelli, cioè, propri dell'otium e della relaxatio animi. ${ }^{16} \mathrm{E}$ in effetti, stando a quanto riferiva l'autore nella conclusio della sua opera, essa traeva origine proprio dalle conversazioni avvenute durante le pause lavorative nelle anticamere del palazzo di papa Martino V tra Poggio stesso e altri importanti segretari papali, delle quali conversazioni il Liber si presentava infatti come fedele registro. ${ }^{17}$

Questo è dunque quanto proponeva il Bracciolini nel suo Liber facetiarum, un testo che stabilirà una volta per tutte le coordinate ideologiche ed estetiche della facezia umanistica, garantendo a essa un prestigio e una diffusione mai ottenuti in precedenza. Ed è in questo clima culturale, nel quale la facetudo, intesa come proprietà necessaria a proiettare sulla corte un'immagine del sé convenevole all'urbanitas e ai culti dei suoi raffinati e illustri convenuti, che va interpretata questa sezione dell'attività "letteraria" di Leonardo da Vinci. In quel contesto, infatti, alla questione, di certo rilevante, della 'buona reputazione', andava spesso a intrecciarsi quella, forse primaria, delle ricche e ambitissime committenze ducali.

La funzione retorico-argomentativa della facezia negli scritti teorici di Leonardo

\footnotetext{
${ }^{12}$ Cfr. nota 6.

13 Petrarca, Rerum memorandarum libri, cit., pp. 140-201.

${ }^{14}$ Quelli previsti, ad esempio, per il dialogo, o ancora per l'epistola familiare di ispirazione ciceroniana, generi orientati a riprodurre su carta le movenze della lingua parlata. Approfondimenti sulla questione si leggono in S. Pittaluga, 'La restaurazione umanistica', in: G. Cavallo, C. Leonardi \& E. Menestò (a cura di), Lo spazio letterario del Medioevo: Il medioevo latino, Vol. II, La circolazione del testo, Roma, Salerno, 1994, pp. 191-217; Id, 'Lorenzo Valla e le Facezie di Poggio Bracciolini', in: M. Regoliosi (a cura di), Lorenzo Valla: la riforma della lingua e della logica. Atti del Convegno del Comitato nazionale, sesto centenario della nascita di Lorenzo Valla, Prato, 4-7 giugno 2008, Firenze, Polistampa, 2010, pp. 191212; A. Bisanti, 'Le "Facezie" di Poggio e la tradizione retorica classica e medievale', in: Critica Letteraria, 22, 4 (1994), pp. 627-653.

${ }_{15}$ P. Bracciolini, Facezie, con un saggio di E. Garin. Introduzione, traduzione e note di M. Ciccuto, Milano, BUR, 1994, pp. 108-111.

${ }^{16}$ Ordine, Teoria della novella e teoria del riso nel Cinquecento, cit., pp. 10-11.

17 Bracciolini, Facezie, cit., pp. 406-409.
} 
Leonardo, omo sanza lettere, sembra in realtà ben consapevole della funzione retorico-argomentativa che le forme della narratio brevis, e in particolar modo la facezia, potevano in quegli anni ricoprire nel contesto del dibattito colto. ${ }^{18} \mathrm{~A}$ quegli umanisti che, ai suoi tentativi di farsi scrittore, dovettero forse rimproverargli una certa inadeguatezza culturale, Leonardo sembrò infatti rispondere indirizzando loro un piccato Proemio giocato proprio attorno a una ironica sentenza:

So bene che, per non essere io litterato, che alcuno prosuntuoso gli parrà ragionevolmente potermi biasimare coll'allegare io essere omo sanza lettere. Gente stolta, non sanno questi tali ch'io potrei, sì come Mario rispose contro a' patrizi romani, io sì rispondere', dicendo: 'Quelli che dall'altrui fatiche se medesimi fanno ornati, le mie a me medesimo non vogliano concedere'. Diranno che, per non avere io lettere, non potere ben dire quello di che voglio trattare. Or non sanno questi che le mie cose son più da esser tratte dalla sperienzia, che d'altrui parola; la quale fu maestra di chi bene scrisse, e così per maestra la piglio e quella in tutti i casi allegherò. (Atl, c. 327v) ${ }^{19}$

In una sorta di conversazione a distanza, Leonardo replicava ai propri morditori in maniera paradossale, rivolgendo contro di loro le loro stesse armi - un apoftegma proveniente dal mondo classico posto qui a sostegno della propria argomentazione per ribaltarne l'opinione e ribadire il primato dell'esperienza sul principio di autorità.

Con quel proemio, Leonardo si inseriva attivamente in una polemica che vedeva allora contrapposti artisti e letterati sul tema del rispettivo primato culturale a corte. ${ }^{20}$ Tale diatriba si era rinfocolata in seguito alla pubblicazione, nel 1490, del volgarizzamento landiniano della Sforziade del Simonetta. ${ }^{21}$ Nell'orazione introduttiva, infatti, l'umanista Francesco Puteolano, primo curatore dell'opera, si era scagliato con forza contro la pittura e la statuaria, riconoscendo alla storia soltanto la facoltà di eternare la memoria dei fatti degli uomini illustri. Punto da tale invettiva, Leonardo avrebbe allora deciso di passare al contrattacco, dando avvio alla realizzazione di un complesso progetto: quello, cioè, di un trattato sulla pittura grazie al quale dimostrare la natura 'mentale' (cioè intellettuale e filosofica) di quell'arte. ${ }^{22}$

\footnotetext{
18 Su questo capitolo dell'attività letteraria di Leonardo utili approfondimenti si leggono in C. Vecce, 'Favole e facezie di Leonardo da Vinci', in: A. Asor Rosa (diretto da), Letteratura italiana. Dizionario delle Opere, Torino, Einaudi, 1999, I, pp. 456-457; Idem, Codex Atlanticus (16). Leonardo: favole e facezie. Disegni di Leonardo dal Codice Atlantico, con la collaborazione di G. Cirnigliaro, Novara, De Agostini, 2013; e, in ultimo, S. Taglialagamba \& M. Versiero, 'Leonardo faceto e grottesco: uno sguardo tragico e comico sulla vita del Rinascimento, negli scritti e nei disegni del genio', in: L. Secchi Tarugi (a cura di), Comico e tragico nella vita del Rinascimento. Atti del convegno internazionale di ChiancianoPienza, 17-19 luglio 2014, Firenze, Cesati, 2016, pp. 433-445.

19 L. da Vinci, Scritti, C. Vecce (a cura di), Milano, Mursia, p. 191.

20 Sugli ambienti umanistici e letterari milanesi all'epoca del Moro imprescindibile è ancora oggi il saggio di E. Garin, 'La cultura a Milano alla fine del Quattrocento', in: Milano nell'età di Ludovico il Moro. Atti del Convegno internazionale (Milano, 28 febbraio - 4 marzo 1983), Milano, Comune di Milano-Archivio storico civico e Biblioteca Trivulziana, 1983, vol. I, pp. 21-28. Aggiornamenti sulla questione, con importanti osservazioni anche sul ruolo della letteratura fiorentina a corte si leggono in S. Albonico, 'Appunti su Ludovico il Moro e le lettere', in: Luisa Giordano (a cura di), Ludovicus Dux, Vigevano, Diakronia, 1995, pp. 66-91. Sui complessi e talora difficili rapporti tra umanisti e potere ducale si veda invece G. Resta, 'La cultura umanistica a Milano alla fine del Quattrocento', in: Milano nell'età di Ludovico il Moro, cit., vol. I, pp. 201-214.

${ }^{21}$ Notizie utili sul volgarizzamento landiniano si leggono in N. Maraschio, 'Lingua, società e corte di una signoria padana fra Quattro e Cinquecento', in: C. Segre (a cura di), Ludovico Ariosto: lingua, stile $e$ tradizione. Atti del congresso (Reggio Emilia-Ferrara, 12-16 ottobre 1974), Milano, Feltrinelli, 1976, pp. 29-38.

22 Questa l'autorevole ipotesi di C. Dionisotti, per la quale cfr. Idem, 'Leonardo uomo di lettere', in: Italia Medioevale e Umanistica, 5 (1962), pp. 183-216, e in part. le pp. 208-215. Sul carattere filosofico della pittura, intesa come strumento di conoscenza, si veda anche l'Introduzione' di Carlo Pedretti a L. Da Vinci, Libro di pittura, Firenze, Giunti, 1995, I.
} 
Colpisce che, in un passaggio chiave di quel testo, il cosiddetto Paragone, dove in discussione era il primato della vista sull'udito, i termini di quella polemica venissero richiamati e in qualche modo risolti ancora una volta proprio attraverso il ricorso a una facezia:

Portando il dì del natale del re Mattia un poeta un'opera fattagli in laude del giorno ch'esso re era a benefizio del mondo [nato], et un pittore gli presentò un ritratto della sua innamorata, subito il re rinchiuse il libro del poeta, e voltossi alla pittura, et a quella fermò la vista con grande ammirazione. Allora il poeta forte isdegnato disse: ' $O$ re, leggi, leggi, e sentirai cose di maggior sustanzia che una muta pittura'. Allora il re, sentendosi riprender del risguardare cose mute, disse: ' $O$ poeta, taci tu, che non sai ciò che ti dica; questa pittura serve a miglior senso che la tua, la quale è da orbi'. (Vat. Urb. Lat. 1270, c.14v) (23 $^{23}$

Sia che se ne mettessero in dubbio le capacità di scrittore, sia che, nelle pagine del Libro di pittura, si discutesse del primato tra parola e immagine, la facezia sembra dunque essere l'arma prediletta da Leonardo per chetare gli 'squilli' dei cosiddetti 'trombetti' di corte, ${ }^{24}$ di quegli 'storiografi, o poeti o altri matematici', cioè, cui era polemicamente indirizzato quel trattato. ${ }^{25}$

Del resto, che Leonardo riconoscesse alla facezia un elevato prestigio culturale appare anche testimoniato dal fatto che, nel tentativo di dotarsi di capacità espressive e stilistiche assimilabili a quelle dei litterati ${ }^{26}$ egli si fosse affidato, come fonte per la pratica del vocabulizare, proprio alle facezie di Poggio, benché traducte de latino in vulgare ornatissimo: a un testo, cioè, che, come si è visto, mirava proprio a esaltare il ruolo della facezia nel contesto della conversazione informale colta. ${ }^{27}$

La vicenda aveva, a ogni modo, davvero del paradossale. Solo pochi decenni prima, infatti, Poggio si era servito di termini provenienti dal fiorentino di uso comune allo scopo di ampliare le possibilità espressive del latino coevo. Ora Leonardo, sulla falsariga di certe prescrizioni landiniane relative all'opportunità di rifarsi al lessico latino per arricchire quelle del toscano, individuava in quella raccolta una fonte dalla quale selezionare vocaboli utili a conferire maggiore dignità espressiva al proprio linguaggio. Nella scrittura, certo, ma forse anche nella conversazione quotidiana.

\section{Leonardo lettore di Poggio Bracciolini e la pratica del vocabulizare}

La presenza del Liber di Poggio negli scaffali della biblioteca di Leonardo era nota da tempo agli esperti di cose vinciane. Il Maestro, infatti, ne aveva registrato il possesso in una celebre lista di libri oggi conservata nel Codice Atlantico (c. $559 r$ ). ${ }^{28}$ Tuttavia, l'invito degli studiosi a rifiutare ogni ipotesi di identificazione tra i volumi menzionati nelle carte leonardesche e l'effettiva cultura del Maestro che non risultasse fondata

\footnotetext{
${ }^{23}$ Da Vinci, Libro di pittura, cit., p. 151.

24 Idem, Scritti, cit., p. 189.

25 Idem, Libro di pittura, cit., p. 143.

26 Tentativo di cui, come è noto, restano abbondanti tracce nelle lunghe liste di vocaboli latini stilate dall'artista negli anni 1487-90, oggi conservate nel cod. Trivulziano 2162. Sull'argomento la bibliografia è, ovviamente, molto vasta. Si rinvia, per essa, a quella, esaustiva, raccolta nella recente edizione delle liste leonardesche da B. Fanini, in: Le liste lessicali del codice Trivulziano di Leonardo da Vinci. Trascrizione e analisi linguistica, Firenze, Cesati, 2018, pp. 193-203.

27 Poggius Florentinus, Facetiae, [Milano, Christophorus Valdarfer, 1484 ca], ISTC ip00872450, f. a1. Sul volgarizzamento delle Facezie come fonte delle liste di vocaboli leonardesche, si vedano, risp., A. Tura, 'Edizioni quattrocentine delle Facezie di Poggio in volgare (e una postilla su Leonardo Lettore)', in: Gutenberg-Jahrbuch, 86 (2011), pp. 77-80; e C. Vecce, 'Una nuova fonte del Codice Trivulziano: le Facezie di Poggio', in: Raccolta vinciana, 37 (2017), pp. 105-130. Da quest'ultimo contributo saranno tratte le citazioni al volgarizzamento riportate nelle pagine che seguiranno.

${ }^{28}$ Un'accurata ricognizione della complessa storia critica di questi documenti è in C. Vecce, La biblioteca perduta. I libri di Leonardo, Roma, Salerno, 2017.
} 
su prove in grado di testimoniare una reale e approfondita conoscenza di quei testi, non suggeriva, in mancanza d'altro, di spingersi molto oltre dal riconoscere, in quel rinvio bibliografico, il segno di una generica indicazione di gusto. ${ }^{29} \mathrm{Grazie}$ a un recente studio di Carlo Vecce oggi sappiamo invece che il Liber facetiarum fu spesso tra le mani di Leonardo, il quale, oltre a ricavarne lemmi per le proprie liste, dovette anche meditarne più e più volte la lezione, e in particolar modo quella, fondamentale per le teorie sul genere faceto che vi trasmetteva, riportata nella dottissima praefatio all'opera. ${ }^{30}$ In tal senso, la recente acquisizione, oltre a recuperare un'inedita, importante fonte alla lessicografia vinciana, contribuisce anche ad arricchire di una pagina significativa il capitolo della cultura umanistica di Leonardo, un capitolo che attende ancora in parte di essere scritto.

Dalla lettura del Liber facetiarum Leonardo poteva certo trarre materiali utili per l'inventio - aneddoti, cioè, da far rivivere nelle mondane conversazioni cortigiane o, come si è visto, da inserire con funzione argomentativa nei suoi scritti teorici -; o, ancora, per l'elocutio - vocaboli dotti che, in un impasto tra fiorentino d'uso vivo e grammatica, dovevano contribuire, in accordo con una prescrizione landiniana spesso richiamata dagli studiosi a commento dell'esercizio leonardesco del vocabulizare, proprio a rendere più eloquente il dicitore innalzando il livello del suo sermo. ${ }^{31}$ Attraverso quella lettura Leonardo poteva però anche riscoprire la natura colta della pratica della facezia, legittimata, infatti, come si è visto, dall'esempio degli antichi:

$A^{\prime}$ quali quando che io risponda mi havere già lecto nostri magiori homini doctissimi et di molta prudentia, grandi piacere et summo studio havere hauto in le cosse fabulose et facete, non da essere ripreheso ma più tosto credo de essere commendato appresso di loro. ${ }^{32}$

Che essa, inoltre, poteva svolgere una specifica funzione ristoratrice massimamente lodata dai filosofi dell'antichità:

Certo honesta cossa, et quasi necessaria credo, ciò che sempre nostri magiori sapientissimi homini laudarono, sapere recreare le mente nostre occupate et presse di molte solicitudine, et con piacevole modo di solazo da gravissime cure rilevarle. ${ }^{33}$

Ma soprattutto, da quelle pagine egli poteva ricavare l'idea che il sermo quotidianus, condotto con studio ed equilibrio, non fosse in realtà in contrasto con eloquentia e doctrina:

Forse molti iudicarano cotesta mia excusatione nascere da culpa et difecto de ingegno, con quali io primamente sentirò dove più ornatamente et più politamente elli cotale cossa

\footnotetext{
29 Tale cautela è stata spesso ribadita dagli studiosi negli anni, e ciò principalmente al fine di stemperare l'entusiasmo dei primi commentatori i quali, partendo dai sintetici riferimenti bibliografici trasmessi in quelle liste, si erano spinti a proporre quali fonti del pensiero del genio toscano testi in realtà del tutto fuori dagli orizzonti culturali di un omo sanza lettere.

30 Dalla praefatio, inoltre, il Maestro prelevò non pochi vocaboli colti - confabulatione, prolate, copiosa, opulenta, per citarne solo alcuni. Leonardo selezionò inoltre lemmi ancora dalle facezie 1-69 e 74-138, come rilevato da C. Vecce in 'Una nuova fonte del Codice Trivulziano: le Facezie di Poggio', cit., p. 108. 31 'Ma non sia alcuno che creda non solamente essere eloquente, ma pure tollerabile dicitore nella nostra lingua, se prima non arà vera e perfetta cognizione delle latine lettere. Perché nessuno dubita che ogni sermone è composto di parole e di sentenzie. Le parole sempre sono inette sanza e' precetti oratori, e le sentenzie sono frivole sanza varia dottrina. [...] Ognuno intende come la latina lingua diventò abondante dirivando molti vocaboli greci in quella, così è necessario che la nostra di ricca venga ricchissima, se ogni dì transferiremo in quella nuovi vocaboli tolti da' Romani e faremoli triti appresso de' nostri'. Il passo, tratto dal Proemio alla Commedia di Dante commentata da Cristoforo Landino, è qui citato da Idem, Scritti critici e teorici, R. Cardini (a cura di), Roma, Bulzoni, vol. I, p. 139.

${ }^{32}$ C. Vecce, 'Una nuova fonte del Codice Trivulziano: le Facezie di Poggio', cit., p. 110. Segnalo in grassetto le parole che, secondo il Vecce, Leonardo avrebbe tratto per le proprie liste di vocaboli.

33 Ivi, pp. 110-111.
} 
scriverano, et ad questo fare li voglio confortare, a ciò la lingua latina anchora in legiera et humile cosse a questa nostra età se trova più copiosa et opulenta. Però che assai credo giovare ad eloquentia et docrtina cotale exercitatione, volsio io experimentare sì molte cosse, le quale parea non poterse dire senza grandissime difficultate latinamente, paressano non absurpamente poterse scrivere $\left[. . . .^{34}\right.$

A ogni modo, al di là di quanto sul piano teorico Leonardo poté realmente apprendere dalla lettura di quei testi, su quello pratico diversi sono i testimoni che ci informano delle sue concrete abilità di conversatore piacevole e faceto. Chi lo conobbe in vita sembra infatti quasi non potere fare a meno di segnalare questo aspetto della sua personalità. Ad esempio Matteo Bandello, il quale nel suo Novelliere racconta di averlo incontrato, quando era ancora un giovane novizio, al convento di Santa Maria delle Grazie, e di averlo visto conversare amabilmente col cardinale di Gurk 'di molte cose e in particolare de l'eccellenza de la pittura', oltre che con alcuni gentiluomini lì radunati, cui racconterà poi la novella di Filippo Lippi rapito dai berberi. ${ }^{35}$ Anche Cristoforo Giraldi riporta, per il tramite del più noto figlio Giovanbattista, un episodio accorso alle Grazie: in questo caso, però, della facezia raccontata Leonardo risulterà addirittura il protagonista. ${ }^{36}$ La storia è quella resa celebre dal Vasari, che la riprenderà infatti nell'edizione giuntina delle Vite, relativa ai ritardi di Leonardo nel completamento del Cenacolo, motivati dall'impossibilità di ritrovare un modello adatto per la figura di Giuda - salvo poi indicarne uno potenziale in Vincenzo Bandello, priore del convento e primo artefice dei solleciti per quei ritardi presso il Duca. Con un motto arguto, ricordava Cristoforo, Leonardo era riuscito a sottrarsi alle pericolose pressioni del Moro e ad accrescere al contempo la sua considerazione presso di lui. Lo stesso Vasari, poi, non dimenticherà di sottolineare ancora quanto egli fosse 'tanto piacevole nella conversazione, che tirava a sé gl'animi delle genti'. ${ }^{37}$ Tra i testimoni indiretti, autorevoli sono, oltre al Vasari, l'Anonimo Gaddiano, unico latore di diverse e molto dettagliate notizie, il quale si limita però a ricordare che Leonardo ' $\mathrm{fu}$ nel parlare eloquentissimo'; ${ }^{38}$ e il pittore milanese Giovanni Paolo Lomazzo, che in un brano del suo Trattato dell'arte della pittura, scoltura e architettura, riporterà un divertente aneddoto incentrato proprio sulla capacità del Maestro di intessere brillanti conversazioni facete:

volendo egli una volta fare un quadro di alcuni contadini che avessero a ridere (tutto che non lo facesse poi, ma solamente lo disegnasse) scelse certi uomini, quali giudicò a suo proposito, et avendosigli fatti familiari col mezzo d'alcuni suoi amici, gli fece un convito; et egli, sedendogli appresso, si pose a raccontare le più pazze e ridicole cose del mondo, in modo che e' gli fece, quantunque non sapessero, di che, ridere alla smascellata. D'onde egli, osservando diligentissimamente, tutti i loro gesti con que' detti ridicoli che facevano impresse ne la mente e poi, doppo che furono partiti, si ritirò in camera et ivi perfettamente gli disegnò in tal modo che non movevano meno essi a riso i riguardanti che si avessero mosso loro le novelle di Leonardo nel convito. (Trattato dell'arte della pittura, scoltura et architettura, II, 1) ) $^{39}$

\footnotetext{
${ }^{34}$ Ivi, p. 111; grassetto mio.

${ }^{35}$ M. Bandello, Novelle, E. Menetti (a cura di), Milano, BUR, 2011, Nov. I, 58, pp. 235-246.

36 Vecce, Leonardo, cit., p. 166.

37 G. Vasari, 'Lionardo da Vinci Pittore e Scultore Fiorentino', in: Id., Le Vite de' più eccellenti architetti, pittori, et scultori italiani, da Cimabue, insino a' tempi nostri. Nell'edizione per $i$ tipi di Lorenzo Torrentino, Firenze, 1550, L. Bellosi \& G. Previtali (a cura di), Torino, Einaudi, 1991, vol. II, p. 546.

38 C. Vecce, Leonardo, cit., p. 361. Alcuni studiosi ipotizzano, tuttavia, che l'Anonimo gaddiano abbia conosciuto Leonardo personalmente.

39 Traggo la notizia e il passo da C. Vecce, Codex Atlanticus (16). Leonardo: favole e facezie. Disegni di Leonardo dal Codice Atlantico, cit., p. 13. Ancora il Lomazzo, nei suoi Sogni e Raggionamenti, farà dire a Leonardo: 'Di corpo fui bello e formato, che con la mia aria rasserenavo ogni animo mesto, e con le parolle volgevo al si et al no ogni indurata intencione'. Il passo è in Idem, Leonardo, cit., p. 379 (corsivo mio).
} 


\section{Le facezie cortigiane di Leonardo}

La consuetudine mostrata da Leonardo a registrare sui propri taccuini quei racconti che ascolta o legge in giro sembra dunque motivata proprio da un'idea della facezia intesa come forma colta e decorosa della conversazione cortigiana. Delle ventotto facezie individuate dagli studiosi tra le carte vinciane, inoltre, un buon numero rinvia proprio a situazioni riconducibili alla vita di corte. Era, quello, il contesto dal quale forse le aveva tratte, e anche quello dove più facilmente poteva servirsene.

Una situazione che poteva essere realmente accaduta alla corte del Moro, e che poteva avere visto proprio Leonardo indossare i panni del vir facetus è quella registrata dal Maestro alla c. 34 del cod. Folll:

Uno artigiano andando spesso a vicitare uno signore, sanza altro proposito dimandare, al quale il signore domandò quello che andava facendo. Questo disse che veniva lì per avere de' piaceri che lui aver non potea; perocché lui volentieri vedeva omini più potenti di lui, come fanno $i$ popolari, ma che 'l signore non potea vedere se non omini di men possa di lui: e per questo i signori mancavano d'esso piacere. (Folll $34 v)^{40}$

La facezia che, come è stato scritto, 'allude alla tematica del riconoscimento professionale e sociale di "artigiani" e "artisti" nella società del rinascimento', ${ }^{41}$ vede ancora una volta un signore ricoprire uno dei poli del circuito comunicativo. La sua presenza nel racconto introduce sull'azione un elemento di forte tensione narrativa, dal momento che in nessun'altra circostanza la corretta riuscita della comunicazione appare altrettanto determinante. ${ }^{42}$ Nella facezia, l'artigiano, che si reca a palazzo non per ragioni di carattere professionale, ma per ricevere lustro dalla frequentazione della corte e dei gentiluomini che di solito la animano, viene punto dal signore, il quale, rilevandone come ingiustificata e inopportuna la presenza in quei luoghi, pone il suo interlocutore in una situazione di notevole imbarazzo. Lo scaltro artigiano, tuttavia, con una rapida e paradossale risposta che, adulando il signore, riesce anche a connotarlo come arguto ed eloquente, arriva a trarsi abilmente d'impaccio, scongiurando così il gravissimo pericolo dell'irrisione collettiva. La facetudo, che, attraverso la conversazione svela pienamente l'ingegno dell'artigiano, può in questo modo riscattarne l'originaria condizione di inferiorità sociale, rivelandolo così quale degno adepto di quella corte. Da questo punto di vista, essa appare come una sorta di dote trasversale e democratica che consente al cortigiano e al signore di riconoscersi quali membri di una medesima società di spiriti eletti.

Tra le facezie raccolte da Leonardo, diverse sono quelle che celebrano l'arguzia del signore. Come gli artigiani-artisti, anche questi ultimi, come si è visto, potevano vantare un filone a loro interamente dedicato. Nella costruzione letteraria della loro immagine, questo genere consentiva di mettere in risalto l'eloquenza, e, quindi, la sapienza del sovrano anche nella gestione degli affari intra moenia:

Uno povero omo fece intendere a uno usceri d'un gran signore come e' dovessi dire al suo signore, che quivi era venuto suo fratello, il quale avea gran bisogno di parlarli. Il quale usceri, avendo referita tale imbasciata, ebbe comessione di dare l'entrata a tale fratello. Il quale, giunto al cospetto del signore, li mostrò come, essendo tutti discesi dal gran padre Adam, ch'elli era suo fratello, e che la roba era mal divisa, e che lo pregava che cacciassi da lui tanta povertà, perché a gran pena potea vivere di limosine. Allora il signori rispose ch'elli era ben lecito tal

\footnotetext{
${ }^{40}$ Da Vinci, Favole e profezie. Scritti letterari, cit., Fac. 1, p. 24. Sulla questione vedi anche S. Schreiber, 'Leonardo da Vinci nella tradizione dei motti di spirito e delle facezie quattrocentesche', in: Quaderni di Retorica, 2 (1986), pp. 63-68.

${ }^{41}$ C. Vecce, Codex Atlanticus (16). Leonardo: favole e facezie, cit., p. 12.

42 Dalla reputazione presso il signore passava, infatti, in via quasi esclusiva il successo sociale e professionale dei membri di una corte.
} 
richiesta e domandò il tesorieri e feceli donare un soldo. Allora il povero ebbe grande ammirazione e disse che quel non si richiedea a tal fratello. Allora il signore disse ch'egli avea tanti simili fratelli, che a dar tanto per ciascuno, che non li rimanea niente a lui, e che tal soldo era bastante a tal divisione di roba. E così con lecita licenzia lo divise da tal redità. (Mall 126v) ${ }^{43}$

In questa facezia, l'azione si sviluppa attraverso un complesso intreccio di discorsi indiretti che si sciolgono in chiusura in un ingegnoso doppio motto arguto, cosa che conferisce al racconto una sorta di effetto umoristico amplificato. $\grave{E}$, questo, un meccanismo narrativo, che ricorre spesso nei racconti conservati nelle carte vinciane. Ne è un esempio ancora, quello, di ascendenza classica, che si legge a c. 45r del Codice Atlantico:

Uno vede una grande spada allato a un altro e dice: 'O poverello! Ell'è gran tempo ch'io t'ho veduto legato a questa arme: perché non ti disleghi, avendo le mani disciolte e possiedi libertà?'. Al quale costui rispose: 'Questa è cosa non tua, anzi è vecchia'. Questo, sentendosi mordere, rispuose: 'lo ti conosco sapere sì poche cose in questo mondo, ch'io credevo che ogni divulgata cosa a te fussi per nova'. (Atl $45 \mathrm{cr})^{44}$

E non aveva torto, qui, il povero gabbato, a rimproverare al suo mordace 'amico' la vetustà di quella battuta: essa, infatti, risaliva addirittura a Macrobio (Saturn., II, 3, 3), ma è probabile che, attraversando i secoli, essa fosse giunta al tardo ' 400 per il tramite di Francesco Petrarca, che l'aveva infatti attinta da quella fonte, inserendola, insieme a molte altre, nei suoi Rerum memorandarum libri. ${ }^{45} \mathrm{Di}$ una sua circolazione negli ambienti umanistici e cortigiani ne lasciava ancora traccia Ludovico Carbone, che la registrava infatti nella sua raccolta. ${ }^{46}$ Come Petrarca, però, l'umanista rimaneva fedele alla lezione originaria, attribuendola a Cicerone e limitando lo scambio di battute a un singolo motto arguto.

Ancora una facezia caratterizzata dalla tematica colta e dalla replicazione del motto arguto è quella registrata da Leonardo alla C. $58 \mathrm{v}$ del cod. $M .{ }^{47}$ Essa mette in scena una conversazione tra uomini dotti intenti a dibattere sul complesso tema della metempsicosi. L'ambientazione ricorda il 'venerabile duello' tenutosi nel 1498 alla corte di Ludovico il Moro, al quale lo stesso Leonardo, stando a quanto riferisce Luca Pacioli nella lettera diretta al Duca che apre la Divina proportione, aveva preso parte: ${ }^{48}$

Uno, volendo provare colla alturità di Pitagora come altre volte lui era stato al mondo, e uno non li lasciava finire il suo ragionamento, allor costui disse a questo tale: 'E per tale segnale che io altre volte ci fussi stato, io mi ricordo che tu eri mulinaro'. Allor costui, sentendosi mordere colle parole, gli confermò essere vero, che per questo contrassegno lui si ricordava che questo tale era stato l'asino, che li portava la farina. (M 58v $)^{49}$

Il raccontino, che fornisce una limpida testimonianza di come una certa facetudo fosse gradita non soltanto nel contesto delle conversazioni cortigiane a sfondo mondano, ma anche in quelle riservate alla discussione di temi dal carattere ben più impegnato, consente anche di aprire una breve parentesi sullo stile di tali ioca leonardeschi. Qui

\footnotetext{
${ }^{43}$ Da Vinci, Favole e profezie. Scritti letterari, cit., Fac. 24, p. 29.

44 Ivi, Fac. 3, p. 24.

45 Petrarca, Rerum memorandarum libri, cit., p. 148.

${ }^{46}$ Carbone, Centotrenta novelle o facezie, cit., Fac.105, p. 73.

47 Paris, Institute de France, cod. M (2183).

48 Luca Pacioli, Divina proportione, Venezia, Alessandro Paganini \& Paganino Paganini imprimebat, 1509, f. 1.

49 Da Vinci, Favole e profezie. Scritti letterari, cit., Fac. 19, p. 28.
} 
sembrano infatti convergere alcuni dei tratti più caratteristici di questa vena della produzione letteraria di Leonardo. La narrazione, che si sviluppa attraverso un rapido botta e risposta con alternanza di discorso diretto e indiretto, è aperta da un pronome indefinito riferito al soggetto faceto, che resta qui privo di ogni altra specificazione; al pronome segue, poi, una proposizione retta da un gerundio 'sospeso', un tratto, questo, caratteristico della scrittura letteraria tardo quattrocentesca, spesso rilevato dagli studiosi nella prosa di Leonardo. ${ }^{50}$ Un verbum dicendi introduce il primo motto, espresso in forma diretta, mentre il secondo, ancora aperto da un verbum dicendi, è riportato qui, come si è detto, indirettamente. Ai poli di questo schema per così dire 'medio', si registrano facezie dal più ampio respiro narrativo, ${ }^{51}$ e altre che constano di un unico scambio di battute, come la seguente, sul tema stranezza-bruttezza:

Uno disse a un altro: 'Tu hai tutti li occhi mutati in istran colore'. Quello li rispose: 'Egli è perché i mia occhi vedono il tuo viso strano'. (Atl 207v) ${ }^{52}$

La facezia in questione, seguita da un'altra ancor più breve che propone una variazione sul medesimo tema, è a sua volta riduzione di un racconto registrato appena sopra, segno del fatto che Leonardo stesse qui puntando a ottenere da quell'aneddoto un effetto di massima concentrazione espressiva.

Tutte, a ogni modo, risultano registrate sul verso di un foglio, datato da Leonardo al 1490, sul quale il Melzi, il dotto allievo di Leonardo che ne ereditò le carte, appuntò: 'Notta ogni cosa. Facetie'. Sul recto, ancora, si trovavano raccolte insieme favole, scritti contro il principio di autorità, informazioni di botanica, registrate da Leonardo con grande cura sia nella grafia che nell'impaginazione. Anche di questi testi il Melzi ritenne necessario tenere memoria, annotando, nuovamente: 'Significationi notta'. Nel loro insieme, questi dati sono stati letti dagli studiosi come segnali di un tentativo di riordino complessivo delle favole e delle facezie, in vista, forse, di una loro pubblicazione autonoma. ${ }^{53}$ Un progetto che presupponeva, dunque, ancora una volta, sia la fiducia nelle proprie capacità di scrittore, sia soprattutto il riconoscimento del valore letterario di quelle forme brevi.

Un'annotazione simile alle due appena viste ('Facietia'), di mano cinquecentesca, si ritrova ancora su un foglio, ricco di scritti letterari, che ha tutto l'aspetto della 'copia in bella', il 327 del C.A., datato dagli studiosi al $1490 .{ }^{54}$ Sul verso, raccolti su due colonne, figurano alcuni tra i più celebri proemi di Leonardo; ${ }^{55}$ sul recto, invece, ben ordinati sulla colonna di destra, campeggiano una favola e due facezie. Una di queste è la cosiddetta Facezia della lavandara, che Leonardo in effetti aveva tratto da un foglio del 1487, modificandone inoltre il titolo da Risposta faceta a Facetia. Con la nuova titolazione, il racconto veniva ricondotto entro l'ambito di un

\footnotetext{
50 Sulle costruzioni paraipotattiche nella prosa di Leonardo si è soffermato A. Marinoni ne Gli appunti grammaticali e lessicali di Leonardo da Vinci, Milano, Castello Sforzesco, 1944, vol. I, pp. 158-161; e ancora in L. da Vinci, Scritti Letterari, Milano, BUR, 1974, pp. 41-47 (con rif. specifico a questa facezia a p. 45). Sulla questione si veda anche G. Ghinassi, 'Casi di "paraipotassi relativa” in italiano antico', in: Studi di grammatica italiana, I (1971), pp. 45-60 e M. L. Altieri Biagi, 'Sulla lingua di Leonardo' in: Ead., Fra lingua scientifica e lingua letteraria, Pisa-Roma-Venezia-Vienna, Istituti editoriali e poligrafici internazionali, 1998, pp. 77-81.

51 Lunghe e articolate sono, ad esempio, le Fac. nn. 16 e 23, che si leggono in Da Vinci, Favole e profezie. Scritti letterari, cit., risp. alle pp. 26-27 e 28-29.

52 Ivi, Fac. 6, pp. 24-25.

53 Vecce, Codex Atlanticus (16). Leonardo: favole e facezie. Disegni di Leonardo dal Codice Atlantico, cit., p. 26.

54 Ivi, p. 30

55 Tra i quali quello che ospitava lo pseudo-apoftegma di Mario, del quale si è discusso poco sopra.
} 
genere ben preciso, segno del fatto che Leonardo fosse ben 'consapevole di inserirsi in una tradizione letteraria'. ${ }^{56}$

Ritornando brevemente alla questione dello stile delle facezie di Leonardo, altra caratteristica degna di nota è che esse risultano quasi sempre prive di ogni elemento riferibile all'identità dei personaggi. In tal senso, esse contravvengono a una sorta di regola non scritta del genere novellistico, che prevede appunto la specificazione dell'identità sociale degli attori coinvolti nella narrazione - nome, luogo di origine, professione -, cosa che consente al narratore di ancorare il suo racconto a uno specifico contesto e di presentarlo come un episodio reale di cronaca municipale. Il fatto è forse riconducibile alla natura modulare di questi appunti, che potevano in tal modo essere rifunzionalizzati da Leonardo a seconda del contesto in cui intendeva riutilizzarli. Le uniche due facezie che trasmettono l'identità dei loro autori - lo pseudo-apoftegma di Mario, e il motto arguto di Mattia Corvino - sono di fatto inscritte in ben riconoscibili contesti letterari.

Poche eccezioni a questa "regola" si possono registrare limitatamente allo status civile dei personaggi faceti - nobiluomini, chierici -, o alla loro professione, nel caso specifico dei pittori. Ne è un esempio la facezia, ancora sul tema della bruttezza fisica, registrata al f. $58 \mathrm{v}$ del cod. $M$ - il medesimo che ospita il 'dialogo sulla metempsicosi' -, dove compare corredata di titolo (Facetia) e di alcune croci di rimando:

Fu dimandato un pittore, perché facendo lui le figure sì belle, che eran cose morte, per che causa esso avessi fatti i figlioli sì brutti. Allora il pittore rispose che le pitture le fece di di e $i$ figlioli di notte. $(M 58 \mathrm{~V})^{57}$

Anche questa facezia aveva un'origine illustre. Essa, infatti, risaliva ancora ai Saturnalia di Macrobio (II, 10, 10), ma è probabile che giungesse fino al tardo ' 400 attraverso la mediazione di Benvenuto da Imola che, nel suo commento alla Commedia, la aveva attribuita a Giotto, in tal modo apostrofato da Dante nel corso di un'ipotetica conversazione avvenuta tra i due presso la cappella degli Scrovegni. ${ }^{58}$

Ricca di riferimenti colti è, ancora, la facezia con la quale ci avviamo verso la conclusione di questo nostro itinerario tra gli scherzi cortigiani di Leonardo, la n. 28, cioè, dell'edizione Vecce-Cirnigliaro, incentrata su un tema già parzialmente accennato all'inizio di questo paragrafo: quello, cioè, dell'origine sociale del cortigiano e della reputazione che da essa ne consegue presso quegli ambienti. La facezia, che inscena un vivace scambio di battute tra un generico morditore e un omo da bene punto per la sua presunta condizione di figlio illegittimo, sembra rinviare alla reale esperienza biografica di Leonardo:

Uno rimproverò a uno omo da bene che non era legittimo. Al quale esso rispose esser legittimo nelli ordini della spezie umana e nella legge di natura, ma che lui nell'una era bastardo, perch'egli avea più costumi di bestia che d'omo, e nella legge delli omini non avea certezza d'esser ligittimo. (Mall 65r) ${ }^{59}$

Attraverso un dotto rimando all'antropologia aristotelica del Politico, Leonardo allestisce qui una bellissima facezia strutturata a chiasmo attorno alla coppia paraetimologica (e paronomastica) bastardo-bestia. In controluce, si scorge il tema

\footnotetext{
${ }^{56} \mathrm{G}$. Ponte, Leonardo prosatore, Genova, Tilgher, 1976, p. 76.

57 Da Vinci, Favole e profezie. Scritti letterari, cit., Fac. 6, pp. 24-25.

58 L. Rossi Casè, Di maestro Benvenuto da Imola, commentatore dantesco, Pergola, Stab. tip. f.lli Gasperini, 1889, pp. 202-203. Su questa facezia vd. anche Taglialagamba \& Versiero, 'Leonardo faceto e grottesco', cit., pp. 436-437.

${ }_{59} \mathrm{Da}$ Vinci, Favole e profezie. Scritti letterari, cit., Fac. 22, p. 28.
} 
umanistico del fine pedagogico della cultura, il cui scopo primario è quello di educare l'originaria condizione ferina dell'uomo adattandolo in tal modo alla vita associata. All'inelegante osservazione dell'avversario, che segnala con essa tutta la propria distanza dal consesso sociale, l'omo da bene oppone infatti una coltissima battuta sul tema della legge civile quale fatto culturale che identifica l'umano in quanto "animale" politico. Sulla legittimità, poi, secondo quella di natura, sembra alludere facetamente l'omo da bene, nessuno può in realtà mai dirsi certo.

Come in quella, già vista, sulla conversazione tra l'artigiano e il signore, anche qui la facezia interviene a livellare ogni distinzione di carattere sociologico in nome dell'ingegno e dell'eloquenza, dal momento che la cultura elegantemente manifestata attraverso una sagace boutade può riscattare ogni sconveniente originaria condizione sociale.

In conclusione, dietro queste facezie sembrano dunque potersi scorgere i segni di un tentativo di adeguamento, da parte di Leonardo, ai modi colti e raffinati degli intellettuali di corte, dal momento che proprio verso le elaborazioni teoriche umanistiche sembrano convergere i tre approcci dell'artista a tale genere descritti nel presente studio: egli infatti si servirà di facezie in funzione retorico-argomentativa nei suoi scritti teorici; ricorrerà al Liber facetiarum di Poggio, principale testo teorico di quel genere, come fonte dalla quale trarre vocaboli "dotti"; raccoglierà e sarà a sua volta autore di numerose facezie colte o più genericamente riconducibili agli ambienti cortigiani.

\section{Parole chiave}

Leonardo da Vinci, facezia, retorica, conversazione, umanesimo

Cristiano Amendola è research fellow nel progetto ERC SKILLNET (Università di Utrecht). Nel 2018 ha conseguito il dottorato di ricerca presso l'Università di Liegi, con una tesi incentrata sulle strategie retoriche e letterarie nella loro comunicazione epistolare durante (XV-XVI secolo). Durante il percorso di dottorato ha collaborato a Early Modern Letters online (Università di Oxford) e all'edizione digitale delle lettere di Giorgio Vasari (EpistolART, Université de Liège). Attualmente lavora al database Human_IT (Università della Basilicata \& Università di Napoli Federico II), dedicato all'analisi retorico-argomentativa del codice epistolare umanista. Sta inoltre preparando un'edizione critica del manuale epistolografico di Bartolomeo Miniatore, il Formulario di lettere missive e responsive.

Rechtstraat $57 \mathrm{H}$

6221 EG Maastricht (Nederland)

c.amendola@uliege.be 
The facezia in the late 15th century, popular form or refined humanistic genre?

A look into a minor section of Leonardo da Vinci's "literary" production

This article briefly traces the general lines of a complex cultural process that led the facezia to assume, through its connection with humanistic rhetoric, a leading role in the context of refined discursive, social, and cultural practices of the courtly life of the second half of the 15th century. By interpreting the facezia in the double meaning of a literary genre and a 'socialized linguistic act', a little-known aspect of Leonardo da Vinci's cultural production is re-interpreted within this framework: i.e. those works in which the Master played the role of a perfect vir facetus. The articles discusses three ways in which Leonardo seemed to appeal to the theoretical elaborations promoted by the humanists. Firstly, he uses rhetorical-argumentative jokes in his theoretical writings. Secondly, he uses Poggio's Liber facetiarum, of which he was a passionate reader, as a source from which he drew 'learned' words. Lastly, he collects and writes numerous jokes of erudite or courtly character. 